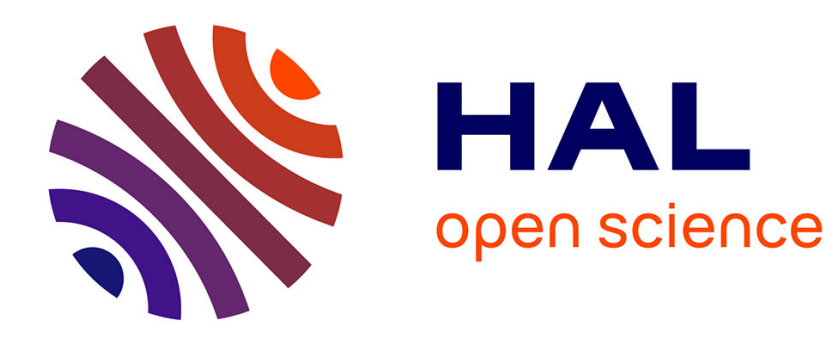

\title{
Unwinding globules under tension and polymer collapse
} Thomas Frisch, Alberto Verga

\section{To cite this version:}

Thomas Frisch, Alberto Verga. Unwinding globules under tension and polymer collapse. Physical Review E : Statistical, Nonlinear, and Soft Matter Physics, 2002, 65, n 4, pp.Art. 041801. 10.1103/PhysRevE.65.041801 . hal-00092022

\section{HAL Id: hal-00092022 https://hal.science/hal-00092022}

Submitted on 8 Sep 2006

HAL is a multi-disciplinary open access archive for the deposit and dissemination of scientific research documents, whether they are published or not. The documents may come from teaching and research institutions in France or abroad, or from public or private research centers.
L'archive ouverte pluridisciplinaire HAL, est destinée au dépôt et à la diffusion de documents scientifiques de niveau recherche, publiés ou non, émanant des établissements d'enseignement et de recherche français ou étrangers, des laboratoires publics ou privés. 


\title{
Unwinding globules under tension and polymer collapse
}

\author{
Thomas Frisch* and Alberto Verga ${ }^{\dagger}$ \\ Institut de Recherche sur les Phénomènes Hors Équilibre, \\ 49, rue F. Joliot-Curie, BP 146, 13384 Marseille, France
}

(Dated: October 24, 2002)

\begin{abstract}
Polymer collapse is known to be mediated by the formation of pearls. These intermediate structures behave as small globules under tension. The globule size is studied by molecular dynamic simulations as a function of the strength of an external stretching force applied to its ends, for different values of the chain length. A very strong first-order-like transition from a compact globule state to a stretched one is observed. A model of this transition in terms of a globule-chain system is presented. The critical force, above which the globule unwinds, is shown to satisfy a power law scaling like $N^{1 / 3}$ in the number of monomers.
\end{abstract}

PACS numbers: 87.15.-v,87.15.Aa,36.20.Ey

Proteins are synthesized as flexible polypeptides and they collapse into a specific compact form called the native form. The kinetics of the folding process is amazingly complex and an understanding of this folding process towards the native state is an important challenge [1-3]. In parallel, stretching experiments on single biomolecules [4] is now a rapidly developing field and may provide information on the elastic behaviour of the molecules that take place during protein folding and give as well some insight into biological function. Even though single homopolymer chain are structurally simpler than proteins, the study of their elastic properties at equilibrium in a bad solvent [5-9], and the study of the dynamical behaviour of the collapse from an extended shape to a compact globule during a rapid quench below the theta point [10-18], are active areas of research. Experimental investigations of the collapse or of the stretching of a homopolymer are sparse due to the enormous difficulty of following the folding of a single chain in a bad solvent at low concentration [19-22]. The picture that has now emerged, however, from these experimental and mainly from numerical studies is that the collapse process, is mediated by the presence of small pearls (a cluster of monomers) linked to one another by a fluctuating stretched chain. Therefore the full dynamics of the collapsing polymer is a complex process which depends in particular on the elastic response of each globule under tension, and on the quench history.

The problem of globule under a stretching force was previously addressed theoretically [5-9] and numerically $[7,9]$. These works showed that the behaviour of a globule under the theta point share some similarities with a first order transition. Below a critical stretching force the globule remains compact, and above the critical force the globule unwinds into a stretched chain. In this Letter we investigate, theoretically and numerically, the mechanisms involved in this first order-like transition, in order

\footnotetext{
*Electronic address: frisch@irphe.univ-mrs.fr
}

${ }^{\dagger}$ Electronic address: verga@irphe.univ-mrs.fr to clarify the origin of the critical force and their scaling properties with respect to the number of monomers. In the first part we describe the collapse from an extended shape to a compact globule using molecular dynamics in the canonical ensemble [23], and we discuss briefly the influence of the quench depth. In the second part, we study a single pearl under tension at equilibrium, and we derive the force-extension relation.

We developed an efficient code to perform off lattice three dimensional molecular dynamics simulations of a homopolymer at finite temperature neglecting hydrodynamic effects. This approach allows us to study very long chains, and to follow their evolution using conservative dynamics in the canonical ensemble. In contrast to other methods there is no need to introduce arbitrary phenomenological parameters on which time scales will depend. We investigated in particular the collapsing transition of a $N=3000$ polymer ( $N$ is the number of monomers), previous studies were conducted by different methods for smaller values of $N$ (Monte-Carlo methods [13, 14, 17], Langevin methods [18]).

The numerical simulations were performed using the Nosé-Hoover method which permits us to work in the canonical ensemble (fixed temperature). The polymer chain contains $N$ molecules interacting through a classical long-range van der Waals force. The connectivity of the chains is respected by using a strong anharmonic potential for neighboring monomers. The Nosé-Hoover equations for the monomer $i$ are

$$
\begin{aligned}
\dot{\mathbf{r}}_{i} & =\mathbf{p}_{i} / m_{i} \\
\dot{\mathbf{p}_{i}} & =-\frac{\partial V_{i}}{\partial \mathbf{r}_{i}}-\xi \mathbf{p}_{i}, \\
\tau_{\xi}^{2} \dot{\xi} & =\frac{2 K}{k_{B} T}-(3 N-6) .
\end{aligned}
$$

Here $\mathbf{r}_{\mathbf{i}}$ and $\mathbf{p}_{i}$ are the position and momentum, $\xi$ is the "friction" variable, $T$ the temperature $\left(k_{B}\right.$ the Boltzmann constant), $\tau_{\xi}=2 Q / k_{B} T$ is the time constant of the heat bath $(Q=$ const. $)$, and $K=\sum_{i=1}^{N} p_{i}^{2} / 2 m_{i}$ is the kinetic energy. The total number of degrees of freedom is $3 N-6$ since we subtracted the translation 


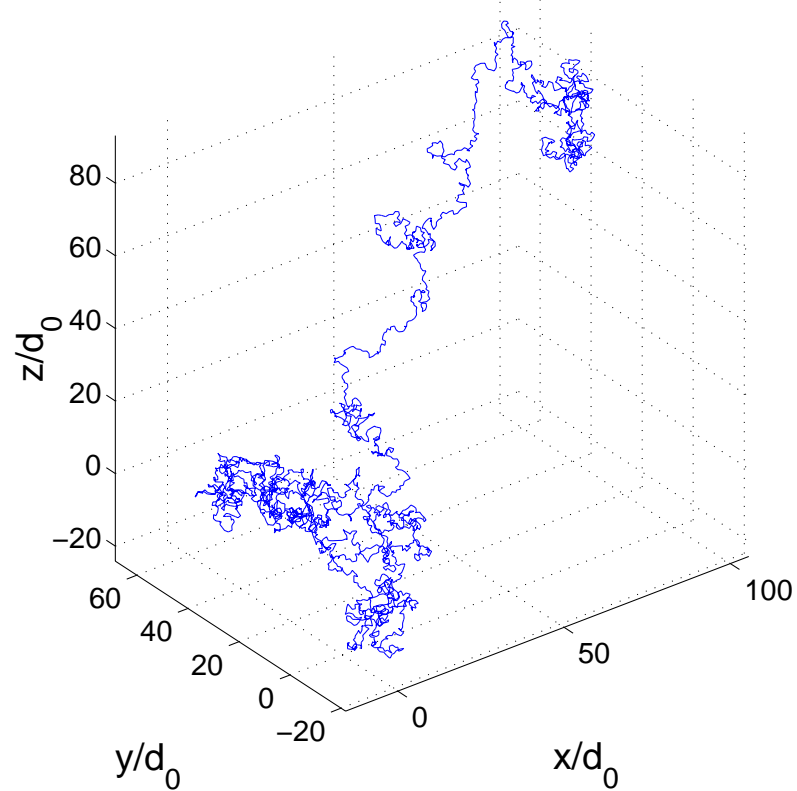

(a)

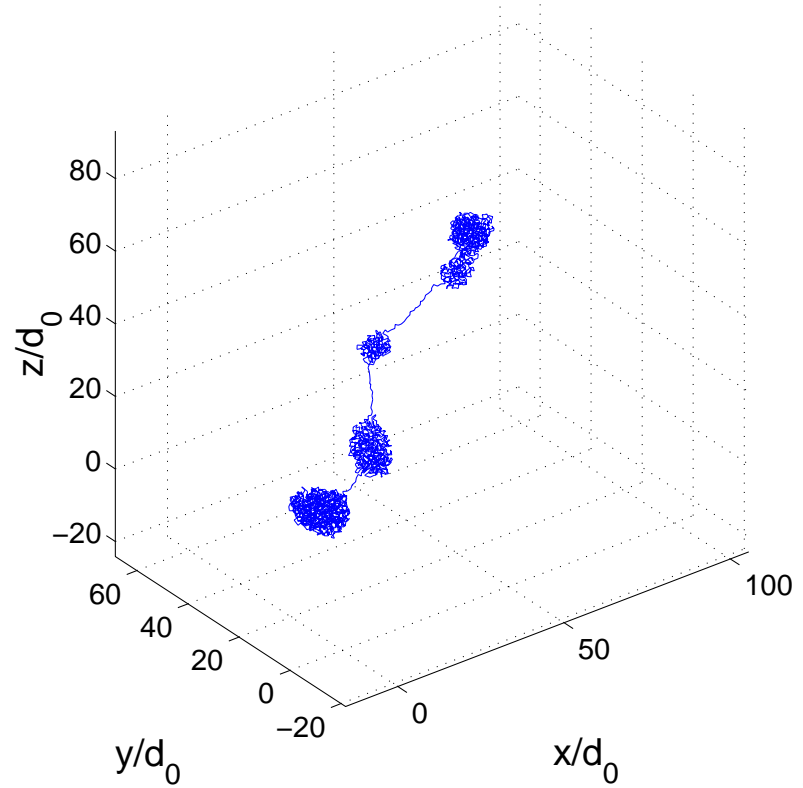

(c)

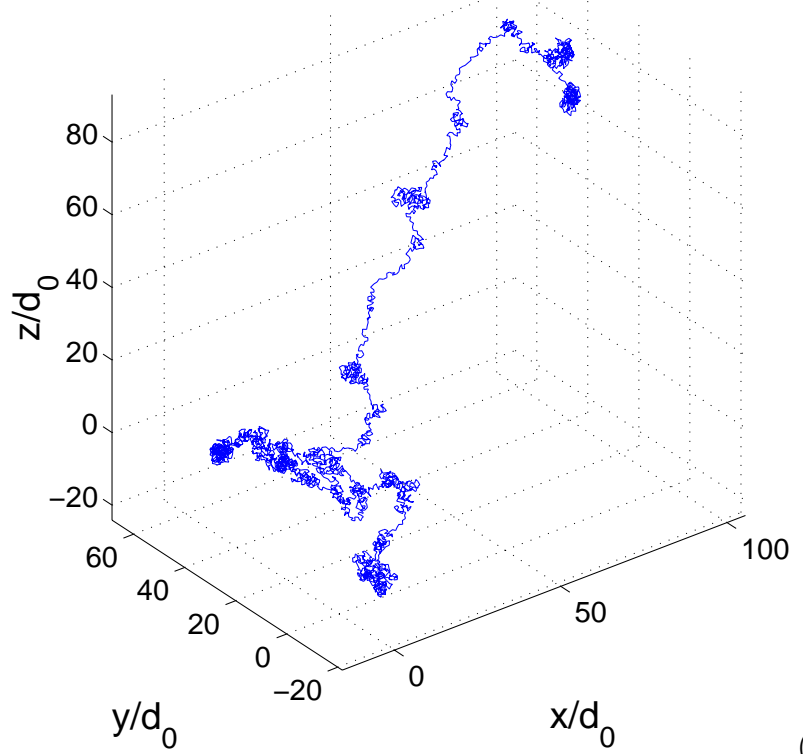

(b)

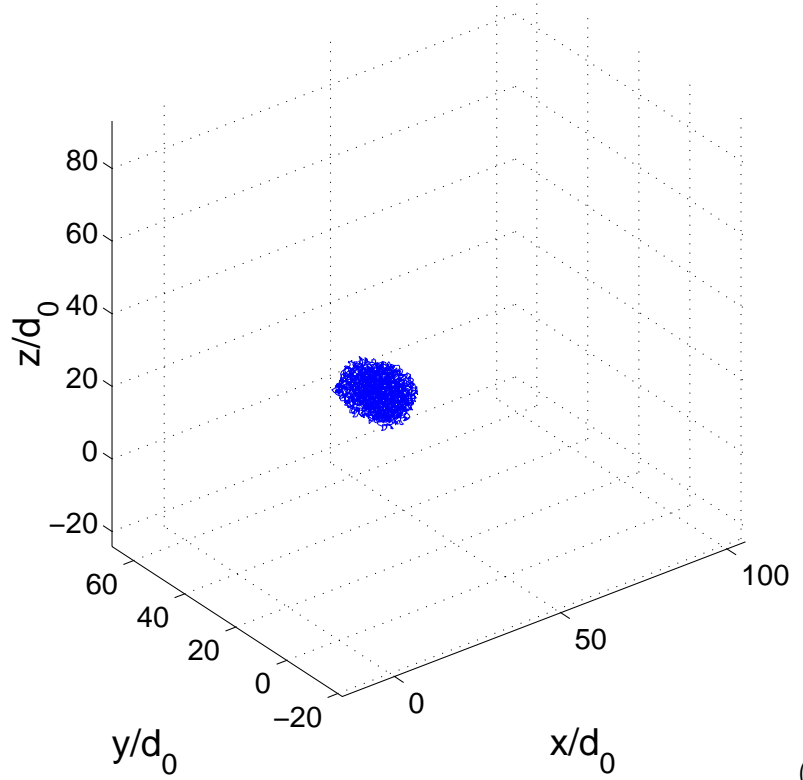

(d)

FIG. 1: Molecular dynamics simulation of a $N=3000$ homopolymer. (a) A self-avoiding random walk generated by a MonteCarlo method $(t=0)$. (b) Nucleation centers appear $\left(t=310^{4} \Delta t\right)$, this state is characterized by a set of pearls separated by stretched chains. (c) Merging of pearls accompanied by a shrinking of the polymer size $\left(t=310^{5} \Delta t\right)$. (d) Globular state $\left(t=610^{5} \Delta t\right)$.

and rotation of the center of mass. Over dots represent time derivatives. The potential $V_{i}$ is the interaction energy of monomer $i$ with all other monomers, it has two contributions $V=V_{1}(r)+V_{2}(r)$ ( $r$ is the distance between monomers): (i) the valence interaction between two neighboring monomers $V_{1}(r)=a\left(r-d_{0}\right)^{2}+b\left(r-d_{0}\right)^{4}$, where $d_{0}$ is the equilibrium distance and $a, b$ are constants characterizing the anharmonic interaction; (ii) the Lenard-Jones interaction between non-neighboring monomers, $V_{2}(r)=\eta\left[(\sigma / r)^{12}-(\sigma / r)^{6}\right]$ where $\eta$ is the potential depth and $\sigma$ the van der Waals radius. Molecular dynamic simulations were performed using an implicit Verlet-Newton-Raphson method for time stepping [23]. The parameters used are: $d_{0}=\sigma=1.0, \eta=0.9$, $a=30, b=100, Q=10$, the time step is $\Delta t=10^{-3}$, and $k_{B} T=0.2$. The $\Theta$-point is about $\Theta \approx 0.7$, as obtained numerically for the chosen parameters. Units are based on $d_{0}$ for lengths, $k_{B} T$ for energies and $\Delta t$ for time.

In the Fig. 1 we present the time evolution of a homopolymer $(N=3000)$ from an initial "swollen coil" 
state to the final (equilibrium) "globule" state. Similar results were obtained by another methods, Monte-Carlo and Langevin simulations, by different groups $[13,14,17]$ The initial state is a self-avoiding random walk modeling a polymer in a good solvent. The gyration radius $R_{g}$ in the swollen coil state scales as $N^{3 / 5}$. At time $t=0$ we quench the polymer below the theta point, in order to trigger the polymer collapse (Fig. 1a). One can distinguish during the collapse different regimes: the initial 'pearl formation' stage, the subsequent stretching of the linking chains accompanied by the pearl growth ("tension regime'), and finally, the pearl 'coalescence regime' which drives the polymer towards the globule state. Among these stages, the tension dominated regime is the longest one. We observe the creation of many pearls which start to grow by adsorbing monomers form the neighboring chains (Fig. 1b). At this stage the total size of the polymer decreases slowly, showing that the clustering is essentially a local process. The interaction between pearls is carried by the linking chains which stretch as pearls grow. Next, the pearls move towards each other and finally coalesce (Fig. 1c). In this regime the strong tension created in the chains leads to a reduction of their thermal fluctuations. The final state consists of a compact globule with a gyration radius scaling as $N^{1 / 3}$ (Fig. 1d). Because of the existence of different regimes, each one having its own time scale, and a typical dependence on the thermodynamical variables $N$ and $T$ [12], one must rule out a simple description of the collapsing process. The different kinetic regimes with their characteristic time scales were extensively discussed in the literature $[12,24]$. These numerical results show that the collapse is largely dominated by the growth of pearls, their properties basically depend on the tension exerted by the neighboring chains. Moreover, each pearl evolves almost independently of others, as long as they are far apart. Therefore, it is interesting to study the behavior of a single pearl under tension, which constitutes a necessary step towards a full description of polymer collapse.

We have also studied the influence of the depth of the quench, here the temperature difference to the theta point. We found that as the quench depth increase, the pearls tends to transform into sausage like objects, which were predicted theoretically by de Gennes [10]. A detailed account of this result will be published elsewhere, but the main effect can be explained in terms of the competition between the kinetic energy (slowing down of monomer mobility) and neighboring monomer attractive interactions.

In order to reproduce the behavior of a single pearl we use a short chain, $N<500$ typically, and we apply to its ends a constant tension $f$. We use as initial condition a well equilibrated globule (Fig. 1d). The force applied to both ends is equal in magnitude and of opposite signs. We measure the mean end-to-end distance $L$, and the mean gyration radius $R_{g}$ as a function of the force. The mean quantities are obtained by temporal averaging over large times at equilibrium. The results of
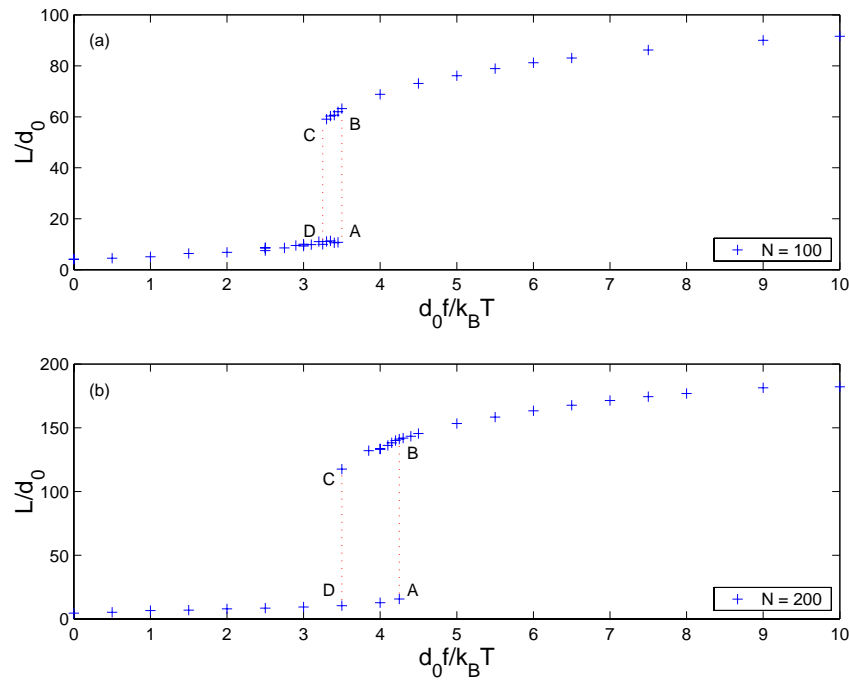

FIG. 2: End-to-end distance as a function of the tension $L=$ $L(f)$. (a) $N=100$; (b) $N=200$. The points A, B, C, and D show the hysteresis cycle.

Fig. 2 show that two well defined states can be identified: a low tension state, for which the globule is only slightly deformed; and a high tension state for which the original pearl is stretched into a chain. Figure 3 shows the typical configuration of the polymer in the initial state, and below and above the critical force. As we increase the tension from zero the globule deforms elastically until a critical value $f_{A}=f_{B}$ for which the globule is suddenly stretched out (Fig. 2). Above $f_{A}$ the system, a stretched coil, is described by the near rod limit. When we decrease the tension, we observe that the length diminishes slowly, passes through the $f_{B}$ point, and at $f_{C}=f_{D}$ jumps back to the globule state. As $f_{C}$ is smaller than $f_{A}$ the transition between the two states is first-order-like and we have a hysteresis cycle.

Another quantity of interest is the gyration radius, which characterizes the globule size, and follows the same behavior as $L$. We observe (Figs. 3ab, and 4) that the globule, in response to a small tension, slightly swells out. This elastic behavior results from the attractive interaction between monomers. It is dominated by internal energy and not by pure entropic effects. A simple theoretical explanation of this phenomenon is given by a model of a globule in equilibrium with a stretched chain (the globule-chain configuration of Fig. 3b). The globule of $N_{g}$ monomers is described by a free energy,

$$
F_{g}=k_{B} T\left(-\frac{|B| N_{g}^{2}}{R^{3}}+\frac{C N_{g}^{3}}{R^{6}}\right),
$$

where the entropic and surface terms were neglected, $R$ is the globule size, it is related to the globule density $n, R \sim R_{g} \sim n^{-1 / 3} ; B=B(T)$ and $C=C(T)$ are the second and third virial coefficients respectively (up to a geometrical factor); below the $\Theta$ temperature $B<0$ 


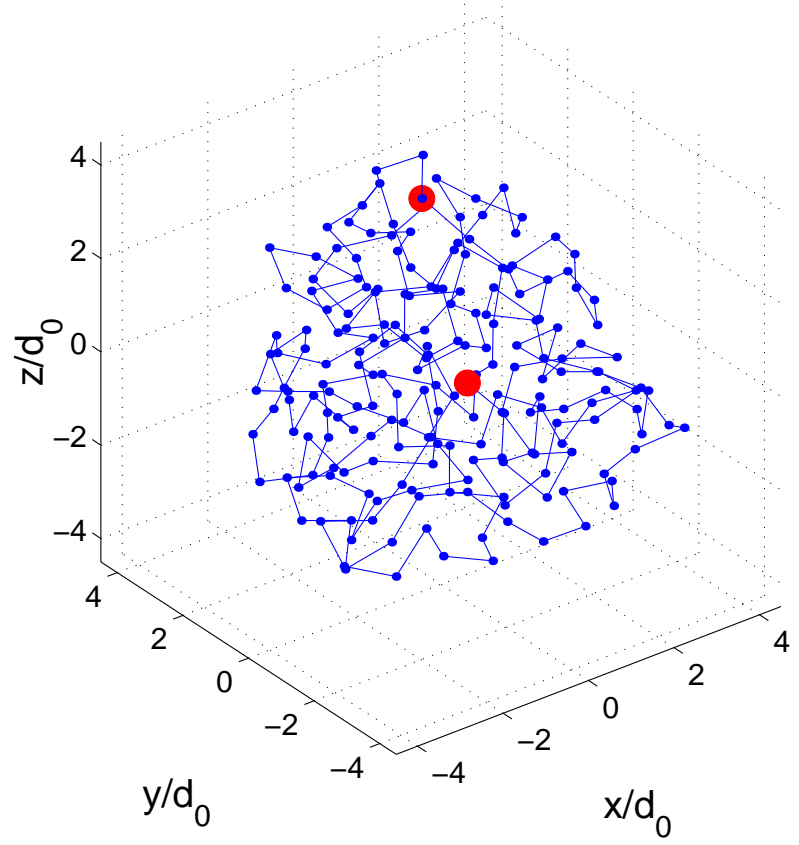

(a)

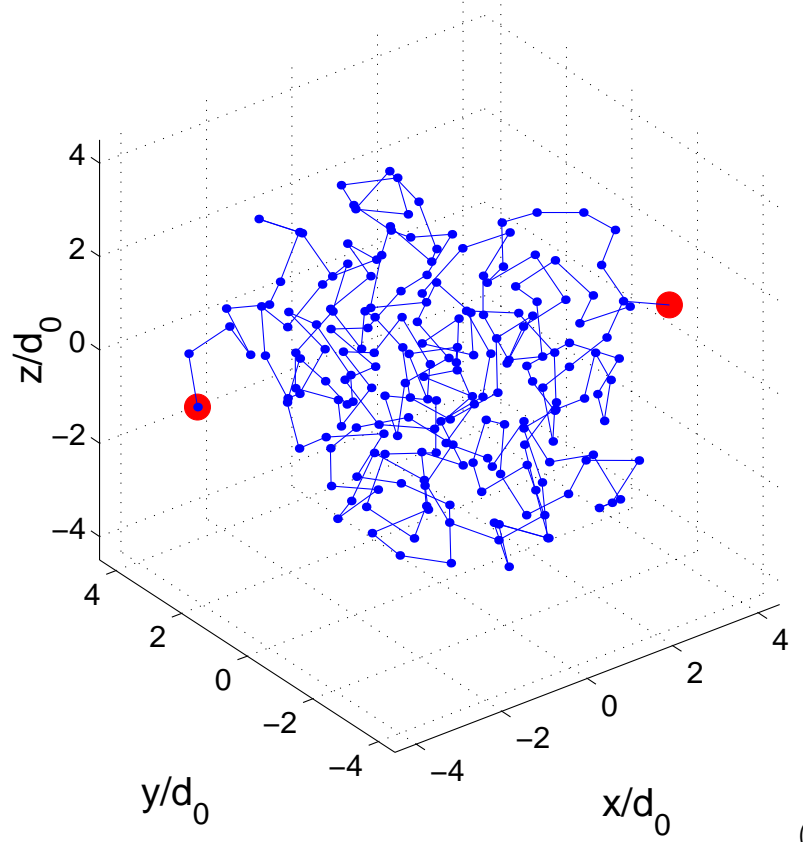

(b)

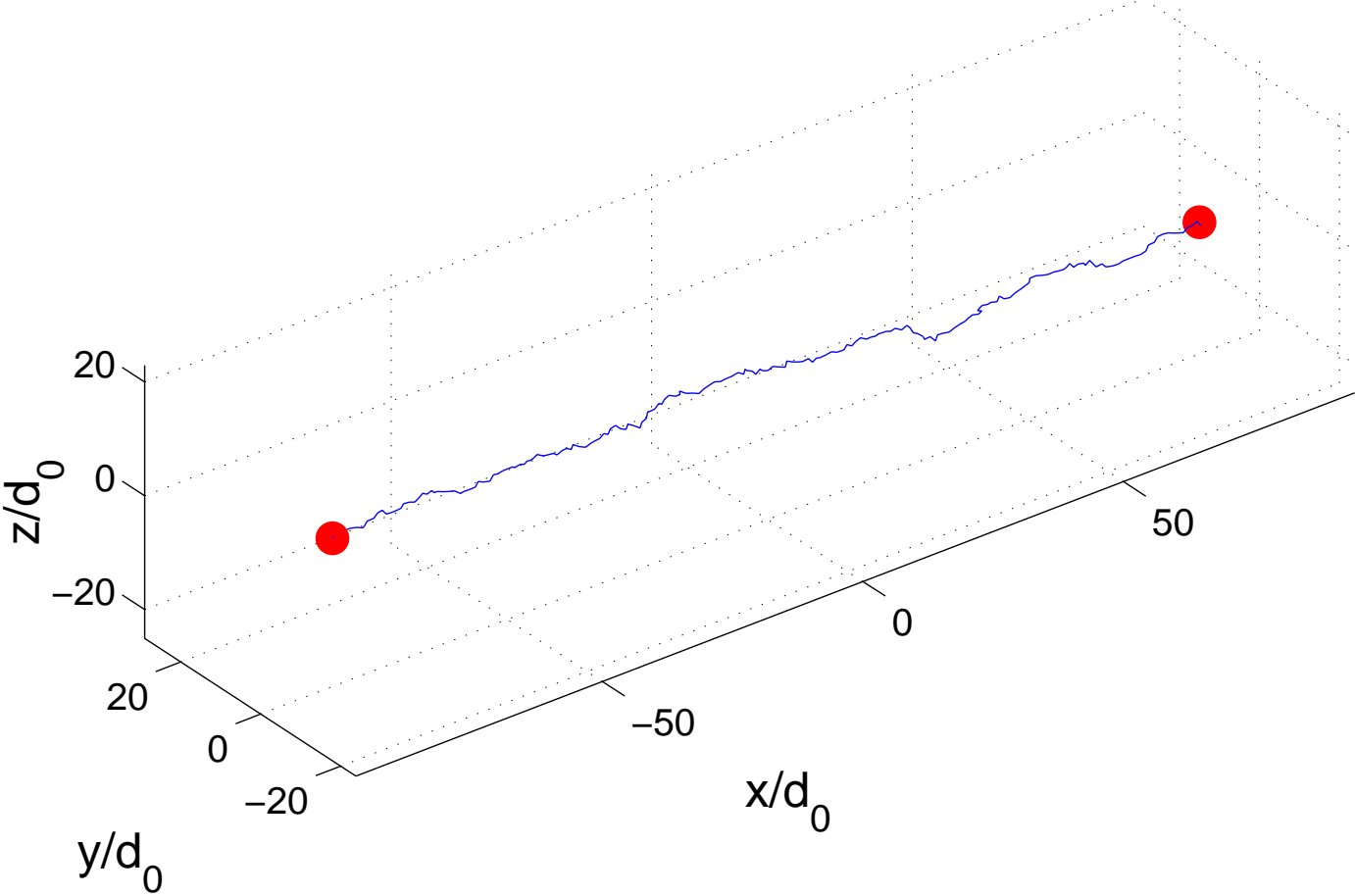

(c)

FIG. 3: Typical configurations of a $N=200$ chain under tension. (a) the globule state for $f=0$; (b) the globule-chain state, below the transition $d_{0} f / k_{B} T=3.5\left(f<f_{A}\right)$; and (c) the stretched chain state for $d_{0} f / k_{B} T=7.5\left(f>f_{A}\right)$.

[25]. In the absence of tension the globule radius is simply given by $R_{e}=\left(2 C N_{g} /|B|\right)^{1 / 3}$. The free energy of a freely joined chain under tension (the configuration of Fig. 3c) is given by the Langevin formula [25],

$$
F_{c}=-k_{B} T N_{c} \ln \left(\frac{4 \pi \sinh y}{y}\right), \quad y=\frac{d_{0} f}{k_{B} T}
$$

where $N_{c}$ is the number of monomers in the chain, and $f$ the applied force; here $F_{c}$ is an implicit function of the chain length $R_{c}=N_{c} d_{0}(\operatorname{coth} y-1 / y)$. When a force is applied to the globule an exchange of monomers with the chain is produced. The equilibrium state is established when the chemical potentials of the globule and the chain are identical. The chemical potential of the globule is: $\mu_{g}-\mu_{g 0}=\left(\partial F_{g} / \partial R\right)\left(\partial R / \partial N_{g}\right)$, where $\mu_{g 0}=\left.\left(\partial F_{g} / \partial N_{g}\right)\right|_{R=R_{e}}=-k_{B} T B^{2} / 4 C$ is the chemical 


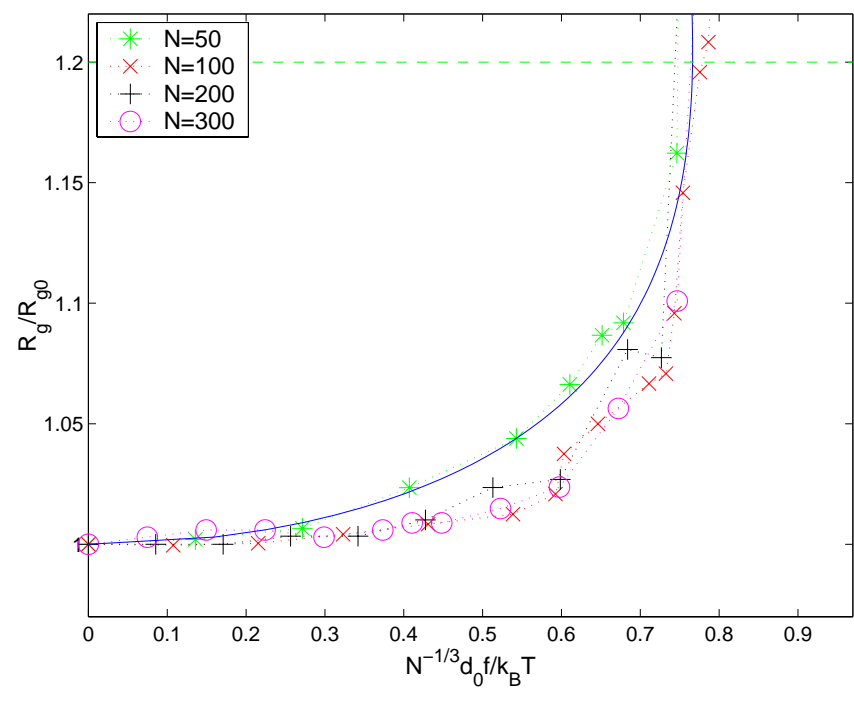

FIG. 4: Mean gyration radius $R_{g} / R_{g 0}$ as a function of the normalized tension $N^{-1 / 3} f / k_{B} T$, for various values of $N$. The solid line is the theoretical result from equation (6) (with $\beta=1.7)$; the horizontal dashed line represent the critical radius $R_{A} / R_{e}=(7 / 4)^{1 / 3}$, and the vertical one is the critical normalized force $f_{A}^{*}$.

potential for $f=0$. Analogously, the chemical potential of the chain is: $\mu_{c}-\mu_{c 0}=\left(\partial F_{c} / \partial R_{c}\right)\left(\partial R_{c} / \partial N_{c}\right)$, where $\mu_{c 0}=\left.\left(\partial F_{c} / \partial N_{c}\right)\right|_{f=0}=-k_{B} T \ln (4 \pi)$. From these two relations, and taking into account that $d N_{g}=$ $-d N_{c}$, one obtains the equilibrium condition between the globule and the chain in the form: $\left(\partial F_{g} / \partial R\right)=$ $-\left(\partial F_{c} / \partial R_{c}\right)\left|d R_{c} / d R\right|$. As the tension is constant along the polymer, the globule density slightly diminishes, while the globule size $R$ increases (see Figs. 3(b) and 4). As long as the number of monomers in the chain satisfies $N_{c} \ll N_{g} \approx N$, the chain-globule interactions are negligible, and one may assume that $\Delta R$ and $\Delta R_{c}$ are proportional: $\left|d R_{c} / d R\right| \equiv 1 / \alpha$, where $\alpha$ is independent of $N_{g}$. Moreover, in the regime of interest (globule-chain coexistence), the tension is large, so that $y \gg 1$. These conditions ensure that the globulechain coupling is weak. From the equilibrium condition, and using that the chain length, for fixed $N_{c}$, is $R_{c}=N_{c} d_{0}(\operatorname{coth} y-1 / y) \approx N_{c} d_{0}(1-1 / y)$, one obtains,

$$
N^{-1 / 3} \frac{d_{0} f}{k_{B} T}=\beta\left[\left(\frac{R_{e}}{R}\right)^{4}-\left(\frac{R_{e}}{R}\right)^{7}\right]^{1 / 2},
$$

where $\beta=\left(3 \alpha d_{0}|B| N^{4 / 3} / R_{e}^{4}\right)^{1 / 2}$, is independent of $N$ since $R_{e} \sim N^{1 / 3}$. To fit the numerical results with (6) we used $\beta=1.7$. From this value of $\beta$ one can compute the free parameter $\alpha$, which is found to be of order one $\alpha=0.4$.[26]

Equation (6) describes the elastic properties of the globule-chain system. Taking into account that the globule radius varies as $R_{e} \sim N^{1 / 3}$, the tension on the globule satisfies $f \sim N^{1 / 3}$ in contrast to the $N^{-1}$ behavior of the Gaussian coil. It is worth mentioning that this scaling law of the critical force with respect to the number of monomers was not obtained in previous works [5-9]. This may be due in part because most numerical studies dealt with the end-to-end distance, which is a highly fluctuating quantity.

We represented in Fig. 4 the graph of the $R_{g}=R_{g}(f)$ function (normalized by $R_{g 0}=R_{g}(0)$ ), using the scaling suggested by (6) for various values of $N$. With the $N^{1 / 3}$ scaling, the curves for different $N$ appear to follow a single tendency. In particular, it is worth noting that the normalized critical force, $f_{A}^{*}=N^{-1 / 3} d_{0} f_{A} / k_{B} T$, is the same for all $N$. The theoretical result (6) also predicts a critical force above which the globule cannot exist. At the critical point $d R / d f \sim \infty$ and the relation $R_{g}=R_{g}(f)$ cannot be satisfied. A simple computation gives $d_{0} f_{A}(N) / k_{B} T=0.45 \beta N^{1 / 3}$ for the critical force, and $R_{A} / R_{e}=(7 / 4)^{1 / 3}$ for the corresponding radius of the globule. These theoretical results are in good agreement with the numerical results obtained for $N=50$ to $N=300$, in the region of validity of the approximations, near the critical force $(y \gg 1)$.

In conclusion, we have presented a theoretical model for the unwinding of a globule under tension and compared it with numerical simulations. The critical force, above which the globule unwinds, is shown to satisfy a power law $N^{1 / 3}$ in the number of monomers. One immediate consequence of the elastic properties of globules is that two neighboring pearls separated by a well stretched chain, and having different sizes cannot be in equilibrium. The larger pearl exerts an attractive force on the smaller one, inducing a drift of the smaller pearl. Another effect, related to the critical force, is that during the initial stages of collapse, small pearls may easily unwind, if the local value of the tension is larger than the critical value. Furthermore, the process of pearl initial size selection, and the coalescence of small pearls into larger ones, may drive the system to a dynamical state characterized by a tension which is throughout near its own critical value.

It would be interesting to study experimentally the influence of the depth of the quench on the collapse. Another interesting issue would be to experimentally measure the force-extension relation, in order to detect the jump in the pearl size (end-to-end distance, gyration or hydrodynamic radius) in large biomolecules, polymers, DNA or proteins, under appropriated conditions.

\section{Acknowledgments}

We acknowledge P. Marcq, J. Palmeri and M. Abid for fruitful discussions. 
[1] T. E. Creigton, Protein Folding (Freeman, New York, 1992).

[2] A. Fersht, Structure and Mechanism in Protein Folding Science (Freeman, New York, 1999).

[3] H. Frauenfelder, P. G. Wolynes, and R. H. Austin, Rev. Mod. Phys. 71(2), S419 (1999).

[4] T. Strick, J. Allemand, D. Bensimon, and V. Croquette, Annual Review of Biophysics and Biological Structure 29, 523 (2000).

[5] A. Halperin and E. B. Zhulina, Macromolecules 24, 5393 (1991).

[6] A. Halperin and E. B. Zhulina, Europhys. Lett. 15, 417 (1991).

[7] M. Wittkop, S. Kreitmeier, and D. Göritz, Phys. Rev. E 53, 838 (1996).

[8] P. Y. Lai, Phys. Rev. E 53, 3819 (1996).

[9] R. G. Maurice and C. C. Matthai, Phys. Rev. E 60, 3165 (1999).

[10] P. G. de Gennes, J. Phys. Lett. (Paris) 46, L639 (1985).

[11] A. Buguin, F. Brochard-Wyart, and P. G. de Gennes, C. R. Acad. Sci. Paris, Série 2 322, 741 (1996).

[12] A. Halperin and P. M. Goldbart, Phys. Rev. E 61, 565 (2000).

[13] B. Ostrovsky and Y. Bar-Yam, Europhys. Lett. 25(6), 409 (1994).

[14] B. Ostrovsky and Y. Bar-Yam, Biophys. Journ. 48, 1694 (1995).

[15] A. Pitard and H. Orland, Europhys. Lett. 41(4), 467
(1998).

[16] E. Pitard and J. P. Bouchaud, Glassy effects in the swelling/collapse dynamics of homogeneous polymers (2001), preprint, http://xxx.lpthe.jussieu.fr/abs/condmat/0010183.

[17] Y. A. Kuznetsov, E. G.Timoshenko, and K. A. Dawson, J. Chem. Phys. 103(11), 4807 (1995).

[18] A. Byrne, P. Kiernan, D. Green, and K. A. Dawson, J. Chem. Phys. 1(1), 573 (1995).

[19] B. Chu, Q.Ying, and A. Y. Grosberg, Macromolecules 28, 180 (1995)

[20] C. Wu and S. Zhou, Phys. Rev. Lett. 77, 3053 (1996).

[21] M. Nakata and T. Nakagawa, Phys. Rev. E. 56, 3338 (1997).

[22] B. Haupt, T. Senden, and E. Sevick, Experimental Evidence of the Rayleigh Instability in Single Polymer Chains (2001), http://www.aps.org/meet/MAR01/baps/abs/G2690009.html.

[23] D. Frenkel and B. Smit, Understanding Molecular Simulations (Academic Press, 1996).

[24] E. G. Timoshenko, Y. A. Kuznetsov, and K. A. Dawson, J. Chem. Phys. 104, 3338 (1996).

[25] A. Y. Grosberg and A. R. Khokhlov, Statistical Physics of Macromolecules (AIP Press, New York, 1994).

[26] The virial coefficient is estimated using the definition $B=(3 / 2) \int\left(1-\exp \left(-V_{2} / k_{B} T\right)\right) r^{2} d r \approx 1.5$, for $\eta=0.9$ and $k_{B} T=0.2$. 